\title{
Holographic subregion complexity in metal/superconductor phase transition with Born-Infeld electrodynamics
}

\author{
Yu Shi, Qiyuan Pan, Jiliang Jing ${ }^{\mathrm{a}}$ \\ Department of Physics, Key Laboratory of Low Dimensional Quantum Structures and Quantum Control of Ministry of Education, and Synergetic \\ Innovation Center for Quantum Effects and Applications, Hunan Normal University, Changsha 410081, Hunan, People’s Republic of China
}

Received: 28 September 2020 / Accepted: 18 November 2020 / Published online: 1 December 2020

(C) The Author(s) 2020

\begin{abstract}
We investigate the holographic subregion complexity (HSC) and compare it with the holographic entanglement entropy (HEE) in the metal/superconductor phase transition for the Born-Infeld (BI) electrodynamics with full backreaction. Based on the subregion CV conjecture, we find that the universal terms of HSC remain finite during phase transitions, and the HSC is a good probe to the critical temperature in the holographic superconducting system. Furthermore, we observe that for the operator $\mathcal{O}_{+}$, the HSC of the superconducting phase decreases first and then increases as the BI parameter increases, which is completely different from that of HEE, and the value of the BI parameter corresponding to the inflection point of HSC is larger than that of HEE. But for the operator $\mathcal{O}_{-}$, the HSC increases monotonically as the BI parameter increases, which is similar to that of HEE.
\end{abstract}

\section{Introduction}

The anti-de Sitter/conformal field theories (AdS/CFT) correspondence [1-4], as a concrete realization of the holographic principle [5,6], provides us a useful theoretical method to study the strongly coupled systems in various fields of physics. In the last decades, two of the most important aspects that have received wide attention in the context of the AdS/CFT correspondence are holographic superconductors [7-10] and holographic entanglement entropy (HEE) $[11,12]$. The former exhibits many characteristic properties shared by the real superconductor, which may be inspiring to understand the mechanism of high temperature superconductors in condensed matter physics, see $[13,14]$ for a review. The latter is a holographic description of quantum entanglement, which might be eventually used to understand the nature of the spacetime geometry.

a e-mail: jljing @ hunnu.edu.cn (corresponding author)
Following the Ryu-Takayanagi (RT) proposal, the entanglement entropy of CFT's states living on the boundary of an AdS spacetime corresponds to the area of a minimal surface defined in the bulk of that spacetime. This is simply summarized into the formula

$\mathcal{S}=\frac{\operatorname{Area}\left(\gamma_{\mathcal{A}}\right)}{4 G_{N}}$

where $\mathcal{S}$ is the entanglement entropy for the subsystem $\mathcal{A}$ which can be chosen arbitrarily, $G_{N}$ is the Newton's constant in the dual gravity theory and $\gamma_{\mathcal{A}}$ known as the RT surface, i.e., the minimal surface in the bulk, which was extended into the bulk with the same boundary $\partial \mathcal{A}$ of subsystem $\mathcal{A}$. Since this proposal provides a simple and elegant way to calculate the entanglement entropy of a strongly interacting system from a weakly coupled gravity dual, it is widely used to study various properties of holographic superconductors at low temperatures [15-29]. These studies show that the entanglement entropy is a good probe to the critical temperature and the order of the phase transition in the holographic superconductor system.

In addition, a new concept which is receving a wide attention of late is the holographic complexity. The (quantum) complexity is an improtant notion in the quantum information theory which has been recently included in the context of holographic field theories. Roughly speaking, the quantum complexity is the minimum number of elementary operations (quantum gates) needed to produce an arbitrary state of interest from a fixed reference state. In the holographic framework, there are two distinct proposals to evaluate the complexity of a holographic boundary state, the first is CV conjecture (complexity $=$ volume) $[30,31]$ and the second is $\mathrm{CA}$ conjecture $($ complexity $=$ action) $[32,33]$. The CV conjecture proposes that the holographic complexity is proportional to the volume of the extremal codimension-one bulk hypersurface which meets the asymptotic boundary on the desired time slice, but the $\mathrm{CV}$ conjecture states that the holo- 
graphic complexity is proportional to the bulk action evaluated in a particular spacetime region known as the WheelerDeWitt patch. Based on the CV conjecture, the definition of holographic complexity for subsystem is given in [34], which shows that the holographic complexity of subsystem $\mathcal{A}$ is proportional to the volume surrounded by the minimal surface (RT surface) as follows

$\mathcal{C}=\frac{\operatorname{Volume}\left(\gamma_{\mathcal{A}}\right)}{8 \pi L G_{N}}$

where $\gamma_{\mathcal{A}}$ is the RT surface of the corresponding subregion $\mathcal{A}$ and $L$ is the AdS radius. This quantity is known as the holographic subregion complexity (HSC). It has been suggested that the possible dual field theory quantity is the fidelity susceptibility in quantum information theory [35-37].

Because the complexity measures the difficulty of turning one state into another, it is expected that the HSC should capture the behavior of the phase transition and can provide useful information as well. In fact, some authors have recently discussed the holographic complexity in different types of holographic superconducting models where the backreaction is taken into account [38-43]. Reference [38] discussed the HSC for two dimensional holographic superconductor with backreactions and argued that extra divergence terms are generated at critical points. However, the numerical results in [39] show that the universal terms of HSC remain finite during the phase transition for one-dimensional holographic superconductors, and the HSC does not behave in the same way as the HEE. Moreover, Ref. [42] has studied the properties of the HEE crossing both first and second order phase transitions in the Stückelberg superconductor, which suggests that the holographic complexity can be probe to the type of superconducting phase transition. It is worth noting that Ref. [43] has investigated the HSC of a $2+1$ dimensional holographic superconductor, which is very similar to the set up in [42] (involves the first order phase transition and second order phase transition), the results of HSC of the superconducting phase during the second order phase transition are opposite to what is found in [42]. For the holographic metal/superconductor phase transition, the HEE in the superconducting case is always less than that in the normal phase [15]. Nevertheless, there are still many ambiguities about the behavior of holographic complexity in holographic superconducting systems. Thus, it is interesting to further analyze the holographic complexity in other holographic superconducting models and to investigate the difference from the HEE.

It's interesting to consider holographic superconductors in the Born-Infeld (BI) electrodynamics. As one of the important nonlinear electrodynamics theories, the BI electrodynamics, which was proposed in 1934 to avoid the infinite selfenergies for charged point particles arising in Maxwell theory, displays good physical properties including the absence of shock waves and birefringence [44-46]. In order to understand the influences of the $1 / \mathrm{N}$ or $1 / \lambda$ ( $\lambda$ is the 't Hooft coupling) corrections on the holographic superconductors, when considering the high order correction of the gauge field, the BI electrodynamics is introduced into the study of holographic superconductors. Researches found that the BI electrodynamics can hinder the formation of the scalar hair so that the holographic superconductor becomes difficult to form $[47,48]$.

In this paper, we would like to investigate the behavior of HSC in the metal/superconductor phase transition with BI electrodynamics, and examine whether the HSC is still useful in describing properties of the phase transition system. We will focus on the time-independent subregion holographic complexity, and compare it to the behavior of HEE. The subregion we choose here is an infinitely long strip with the width $\ell$. We will see that the universal terms of HSC remain finite during phase transitions, just like the HEE, and the critical temperature at which a normal phase turns into a superconducting phase is exactly the same in both the HSC and the HEE computation. However, the behavior of HSC is different from that of HEE. Moreover, for the two operators, the HSC exhibits very different behaviors. In particular, in the superconducting phase, with the increase of the BI factor, the HSC of the operator $\left\langle\mathcal{O}_{-}\right\rangle$increases monotonously but the HSC first decreases and then increases for the operator $\left\langle\mathcal{O}_{+}\right\rangle$.

This paper is organized as follows. In Sect. 2, we will introduce the holographic superconductors in the BI electrodynamics. In Sect. 3, we study the phase transition with BI electrodynamics in four-dimensional AdS black hole spacetime. In Sect. 4, we first review the holographic setup of the entanglement entropy and subregion complexity for a strip subregion, and then numerically evaluate them in the superconducting model. In Sect. 5, we summarize our results.

\section{Holographic superconductor in BI electrodynamics}

The action for the $d$-dimensional gravity and BI electromagnetic field coupling with a charged scalar field can be expressed as

$$
\begin{aligned}
S & =\int d^{d} x \sqrt{-g} \\
& \times\left[\frac{1}{2 \kappa^{2}}(R-2 \Lambda)-|\nabla \Psi-i q A \Psi|^{2}-m^{2}|\Psi|^{2}+\mathcal{L}_{B I}\right],
\end{aligned}
$$

where $g$ is the determinant of the metric, $\psi$ represents a scalar field with charge $q$ and mass $m, \kappa^{2}=8 \pi G_{d}$ is the $d$-dimensional gravitational constant, $\Lambda=-(d-1)(d-$ 2) $/ 2 L^{2}$ is the cosmological constant, $A$ is the gauge field, 
and $\mathcal{L}_{B I}$ is Lagrangian density of the BI electrodynamics

$\mathcal{L}_{B I}=\frac{1}{b^{2}}\left(1-\sqrt{1+\frac{b^{2} F^{\mu \nu} F_{\mu \nu}}{2}}\right)$.

Here $F^{\mu v}$ is the strength of the BI electrodynamic field $F=$ $d A$, and $b$ is the BI coupling parameter. In the limit $b \rightarrow 0$, the BI field will reduce to the Maxwell field. Including the backreaction, we consider the metric ansatz

$d s^{2}=-f(r) e^{-\chi(r)} d t^{2}+\frac{d r^{2}}{f(r)}+r^{2} h_{i j} d x^{i} d x^{j}$.

Then the Hawking temperature of the black hole is expressed as

$T_{H}=\frac{f^{\prime}\left(r_{+}\right) e^{-\chi\left(r_{+}\right) / 2}}{4 \pi}$,

where $r_{+}$is the horizon of black hole. We need $\chi(r \rightarrow \infty)=$ 0 to recover the AdS boundary. The electromagnetic field and the scalar field can be taken as

$A=\phi(r) d t, \quad \psi=\psi(r)$.

From above assumptions, we can obtain the equations of motion as

$$
\begin{aligned}
\psi^{\prime \prime} & +\left(\frac{d-2}{r}-\frac{\chi^{\prime}}{2}+\frac{f^{\prime}}{f}\right) \psi^{\prime}+\frac{1}{f}\left(\frac{q^{2} e^{\chi} \phi^{2}}{f}-m^{2}\right) \\
\psi & =0, \\
\phi^{\prime \prime} & +\left(\frac{d-2}{r}+\frac{\chi^{\prime}}{2}\right) \phi^{\prime}-\frac{(d-2) b^{2} e^{\chi}}{r} \phi^{\prime 3} \\
& -\frac{2 q^{2} \psi^{2}\left(1-b^{2} e^{\chi} \phi^{\prime 2}\right)^{\frac{3}{2}}}{f}=0, \\
\chi^{\prime} & +\frac{4 r \kappa^{2}}{d-2}\left(\psi^{\prime 2}+\frac{q^{2} e^{\chi} \phi^{2} \psi^{2}}{f^{2}}\right)=0, \\
f^{\prime} & -\left(\frac{(d-1) r}{L^{2}}-\frac{(d-3) f}{r}\right) \\
+ & \frac{2 r \kappa^{2}}{d-2}\left[m^{2} \psi^{2}+f\left(\psi^{\prime 2}+\frac{q^{2} e^{\chi} \phi^{2} \psi^{2}}{f^{2}}\right)\right. \\
+ & \left.\frac{1-\sqrt{1-b^{2} e^{\chi} \phi^{\prime 2}}}{b^{2} \sqrt{1-b^{2} e^{\chi} \phi^{\prime 2}}}\right]=0,
\end{aligned}
$$

where the prime denotes the derivative with respect to $r$. At the horizon $r_{+}$, the regularity condition gives the boundary conditions $\phi\left(r_{+}\right)=0$ and $f\left(r_{+}\right)=0$. Near the AdS boundary $(r \rightarrow \infty)$, the asymptotic behaviors of the solutions are

$$
\begin{aligned}
& \chi \rightarrow 0, \quad f \sim r^{2}, \quad \phi \sim \mu-\frac{\rho}{r^{d-3}}, \quad \psi \sim \frac{\psi_{-}}{r^{\Delta_{-}}} \\
& +\frac{\psi_{+}}{r^{\Delta_{+}}},
\end{aligned}
$$

with $\Delta_{ \pm}=\left((d-1) \pm \sqrt{(d-1)^{2}+4 m^{2}}\right) / 2$, where $\mu$ and $\rho$ are interpreted as the chemical potential and charge density in the dual field theory respectively. The coefficients $\psi_{+}$and $\psi_{-}$correspond to the vacuum expectation values $\left\langle\mathcal{O}_{-}\right\rangle$and $\left\langle\mathcal{O}_{+}\right\rangle$of an operator $\mathcal{O}$ dual to the scalar field [8,9].

Considering the Breitenlohner-Freedman bound [49,50], the mass of the scalar field must be restricted as $\mathrm{m}^{2}>$ $-(d-1)^{2} / 4$. In addition, it should be noted that provided $\Delta_{-}$is larger than the unitarity bound, both $\psi_{-}$and $\psi_{+}$can be normalizable where $-(d-1)^{2} / 4<m^{2}<-(d-1)^{2} / 4+1$ [10]. This means that $\psi_{-}$or $\psi_{+}$can either be identified as a source or an expectation value. In the following calculation, we impose boundary condition that either $\psi_{-}$or $\psi_{+}$vanishes. From the equations of motion for the system, we can get the useful scaling symmetries in the forms

$$
\begin{aligned}
& r \rightarrow \alpha r, \quad(x, y, t) \rightarrow(x, y, t) / \alpha, \quad \phi \rightarrow \alpha \phi, f \rightarrow \alpha^{2} f \\
& L \rightarrow \alpha L, \quad r \rightarrow \alpha r, \quad t \rightarrow \alpha t, \quad q \rightarrow \alpha^{-1} q, \\
& e^{\chi} \rightarrow \alpha^{2} e^{\chi}, \quad \phi \rightarrow \alpha^{-1} \phi, \quad t \rightarrow t \alpha .
\end{aligned}
$$

Using the scaling symmetries (13) we can take $r_{+}=1$, and the symmetries (14) allow us to set $L=1$.

\section{Phase transition with BI electrodynamics}

In this part, we concretely consider the four-dimensional AdS black hole spacetime. We will investigate the physical properties of phase transition in this model through the behaviors of the scalar condensation. For the normal phase, the metric becomes the Reissner-Nordström AdS black hole as the BI parameter approaches to zero. Thus, we have

$\chi=\psi=0, \phi=\rho\left(\frac{1}{r_{+}}-\frac{1}{r}\right), f=r^{2}+\frac{\rho^{2}}{4 r^{2}}-\frac{r_{+}^{3}}{r}-\frac{\rho^{2}}{4 r_{+} r}$.

However, if the BI parameter is not equal to zero, the solution is the BI AdS black hole.

For purpose of getting the solutions in the superconducting phase where $\psi(r) \neq 0$, we make a coordinate transformation from $r$-coordinate to $z$-coordinate by defining $z=r_{+} / r$. Then, the equations of motion can be rewritten as

$$
\psi^{\prime \prime}-\left(\frac{\chi^{\prime}}{2}-\frac{f^{\prime}}{f}\right) \psi^{\prime}-\frac{1}{z^{4} f}\left(m^{2}-\frac{e^{\chi} q^{2} \phi^{2}}{f}\right) \psi=0,
$$

$$
\begin{aligned}
& \phi^{\prime \prime}+\frac{1}{2} \chi^{\prime} \phi^{\prime}+2 z^{3} b^{2} e^{\chi} \phi^{\prime 3} \\
& -\frac{2 q^{2} \psi^{2}\left(1-b^{2} e^{\chi} z^{4} \phi^{\prime 2}\right)^{\frac{3}{2}}}{z^{4} f} \phi=0,
\end{aligned}
$$




$$
\begin{aligned}
& \chi^{\prime}-2 \kappa^{2}\left(z \psi^{\prime 2}+\frac{e^{\chi} q^{2} \phi^{2} \psi^{2}}{z^{3} f^{2}}\right)=0, \\
& f^{\prime}-\frac{f}{z}+\frac{3}{z^{3}} \\
& -\frac{\kappa^{2}}{z^{3}}\left[m^{2} \psi^{2}+f\left(z^{4} \psi^{\prime 2}+\frac{1}{f^{2}} e^{\chi} q^{2} \phi^{2} \psi^{2}\right)\right. \\
& \left.+\frac{1-\sqrt{1-b^{2} z^{4} e^{\chi} \phi^{\prime 2}}}{b^{2} \sqrt{1-b^{2} z^{4} e^{\chi} \phi^{\prime 2}}}\right]=0,
\end{aligned}
$$

where the prime now denotes the derivative with respect to $z$. Since the equations are nonlinear and coupled to each other in the superconducting phase, we will solve these equations by using the numerical shooting method. Considering the Breitenlohner-Freedman bound, we set $m^{2}=-2$ and $q=1$ without loss of generality, and we set the strength of backreaction $2 k^{2}=16 \pi G_{4}=1$ in our following study. Since there are scaling symmetries described by Eq. (13) for the equations of motion, the following quantities can be rescaled as

$$
\begin{gathered}
\mu \rightarrow \alpha \mu, \quad \rho \rightarrow \alpha^{2} \rho, \quad\left\langle\mathcal{O}_{+}\right\rangle \rightarrow \alpha^{2} \\
\left\langle\mathcal{O}_{+}\right\rangle, \quad\left\langle\mathcal{O}_{-}\right\rangle \rightarrow \alpha\left\langle\mathcal{O}_{-}\right\rangle .
\end{gathered}
$$

The condensates of the scalar operators as a function of temperature with different values of the BI parameter are shown in Fig. 1. The left panel is the case of condensate $\left\langle\mathcal{O}_{+}\right\rangle$and right panel shows the case of condensate $\left\langle\mathcal{O}_{-}\right\rangle$. It can be easily seen from the pictures that when the temperature is higher than the critical temperature $T_{c}$, there is no condensation and it can be regarded as a metal phase. However, the condensation occurs when the temperature is below the critical temperature $T_{c}$, which corresponds to a superconducting phase. In Table 1, we list the critical temperature for the condensation of two operators for different BI parameters. Obviously, the critical temperature $T_{c}$ decreases as the BI parameter $b$ increases, which means that the effect of the BI correction to the usual Maxwell field is to make it harder for the scalar hair to form. This result is consistent with Ref. [21] and the same as the result in [9] as the BI parameter $b$ approaches to zero.

\section{HEE and HSC of the holographic model}

We consider the subsystem $\mathcal{A}$ with a straight strip geometry which is described by $-\frac{\ell}{2} \leq x \leq \frac{\ell}{2}$ and $-\frac{R}{2}<y<$ $\frac{R}{2}(R \rightarrow \infty)$, where $\ell$ is defined as the size of region $\mathcal{A}$ and $R$ is a regulator which can be set to infinity. In order to use the $z$-coordinates form we choose a time slice in the metric (5) and replace the coordinate $r$ by $r_{+} / z$. Note that we take $r_{+}=1$ there. The hypersurface surface $\gamma_{A}$ starts from $z=\epsilon$ at $x=\frac{\ell}{2}$, then extends into the bulk until it reaches $z=z_{*}$, and returns back to the AdS boundary $z=\epsilon$ at $x=-\frac{\ell}{2}$, where $\epsilon$ is UV cutoff. Therefore, we may obtian the induced metric on the minimal surface as follow

$d s_{\text {induced }}^{2}=\frac{1}{z^{2}}\left\{\left[1+\frac{1}{z^{2} f}\left(\frac{d z}{d x}\right)^{2}\right] d x^{2}+d y^{2}\right\}$.

By using the proposal given by Eq. (1), the entanglement entropy in the strip geometry is

$\mathcal{S}=\frac{2 R}{4 G_{4}} \int_{-\ell / 2}^{\ell / 2} \frac{d x}{z^{2}} \sqrt{\frac{1}{z^{2} f}\left(\frac{d z}{d x}\right)^{2}+1}$.

The minimality condition implies

$\frac{d z}{d x}=\frac{1}{z} \sqrt{\left(z_{*}^{4}-z^{4}\right) f}$,

in which the constant $z_{*}$ satifies the stationary condition $\left.\frac{d z}{d x}\right|_{z=z_{*}}=0$. Integrating the condition gives us

$x(z)=\int_{z}^{z_{*}} d z \frac{z}{\sqrt{\left(z_{*}^{4}-z^{4}\right) f(z)}}$,

which satisfies, with a UV cutoff $\epsilon$,

$x(\epsilon \rightarrow 0)=\frac{\ell}{2}$.

Using Eq. (24), the HEE can be rewritten as

$\mathcal{S}=\frac{2 R}{4 G_{4}} \int_{\varepsilon}^{z_{*}} d z \frac{z_{*}^{2}}{z^{3}} \frac{1}{\sqrt{\left(z_{*}^{4}-z^{4}\right) f(z)}}=8 \pi R\left(s+\frac{1}{\epsilon}\right)$,

where $s$ is a finite term which is physically important and the term $1 / \epsilon$ is divergent.

By using the proposal given by Eq. (2), the HSC in the strip geometry is

$\mathcal{C}=\frac{2 R}{8 \pi L G_{4}} \int_{\epsilon}^{z *} \frac{x(z) d z}{z^{4} \sqrt{f}}=\frac{R}{4 \pi L G_{4}}\left[c+\frac{F\left(z_{*}\right)}{\epsilon^{2}}\right]$,

where $c$ is a universal term and $F\left(z_{*}\right) / \epsilon^{2}$ is a diverging term [39]. Since the value of universal term should not change for different cutoffs, for two different values of cutoff $\epsilon_{1}$ and $\epsilon_{2}$, the value of $F\left(z_{*}\right)$ in different situations can be found numerically. For more details on the regularization of holographic complexity, see [51,52].

Under the scaling symmetries of Eq. (13), we can rescale the $\ell, s$ and $c$ as

$\ell \rightarrow \alpha^{-1} \ell, \quad s \rightarrow \alpha s, \quad c \rightarrow \alpha c$.

Therefore, in the following calculation we can use these dimensionless quantities

$\ell \sqrt{\rho}, \quad \frac{s}{\sqrt{\rho}}, \quad \frac{c}{\sqrt{\rho}}$. 

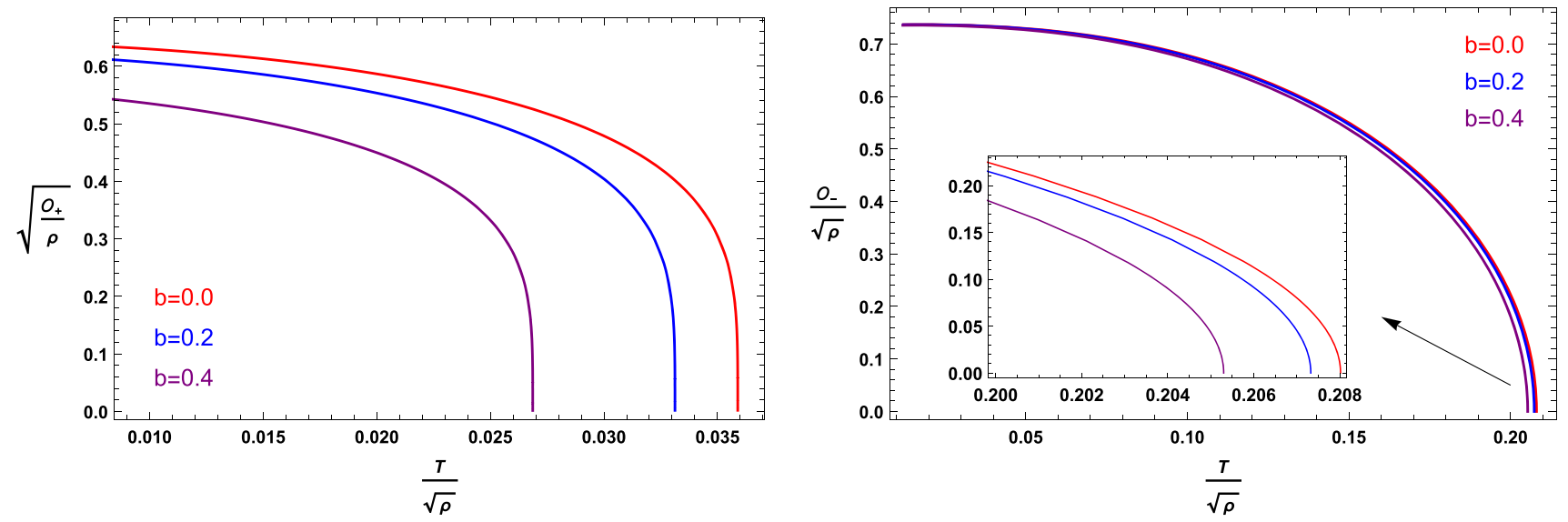

Fig. 1 The condensates of the scalar operators $\mathcal{O}_{+}($left $)$and $\mathcal{O}_{-}$(right) versus temperature for different values of the BI parameter. The colored curves correspond to $b=0$ (red), $b=0.2$ (blue) and $b=0.4$ (purple), respectively

Table 1 The critical temperature for the phase transition with different values of the BI parameter $b$

\begin{tabular}{lllll}
\hline & $\mathrm{b}$ & 0 & 0.2 & 0.4 \\
\hline$\left\langle\mathcal{O}_{+}\right\rangle$ & $T_{c} / \sqrt{\rho}$ & 0.035891 & 0.033127 & 0.026864 \\
$\left\langle\mathcal{O}_{-}\right\rangle$ & $T_{c} / \sqrt{\rho}$ & 0.208017 & 0.207326 & 0.205301 \\
\hline
\end{tabular}

Next, we will study the behaviors of HSC and HEE for two operators numerically.

\subsection{The results of operator $\mathcal{O}_{+}$}

We present the HEE and HSC of the operator $\mathcal{O}_{+}$as a function of temperature $T$ and the BI parameter $b$ in Fig. 2. The left panel is the case of HEE and right panel shows the case of HSC. It can be seen from the right panel that the points, where the curves of HSC for the normal phase (solid) intersect with those of the superconducting phase (dashed), occur at critical temperatures $T_{c} / \sqrt{\rho}=0.035891, T_{c} / \sqrt{\rho}=0.033127$ and $T_{c} / \sqrt{\rho}=0.026864$, for BI parameters $b=0, b=0.2$ and $b=0.4$, respectively. The discontinuous change of HSC at the critical point indicates that the system has undergone a phase transition from a normal state to a superconducting one. Moreover, we can also see that the critical temperature $T_{c}$ of the phase transition decreases as the BI parameter $b$ increases. The information is consistent with the results in left panel, which is obtained by analyzing the behavior of HEE. This means the HSC can be used to search for the critical temperature of the phase transition, just as the HEE.

It can be seen from the left plot in Fig. 2 that the HEE in the superconducting phase is always less than the one in the normal case and drops as the temperature decreases. This property holds for different values of the BI parameter. This behavior of the HEE is due to the fact that the condensate turns on at the critical temperature and the formation of Cooper pairs makes the degrees of freedom decrease in the superconducting phase. However, as shown in right plot of Fig. 2, the values of HSC for the superconducting state is always larger than that for the normal state and increases as the temperature decreases. This result is in agreement with that reported in $[39,43]$.

We also show the behaviors of the HEE and HSC with respect to the BI parameter $b$ for different widths $\ell$ at a fixed temperature $T / \sqrt{\rho}=0.015$ below the phase transition temperature in Figs. 3 and 4 respectively. Obviously, for a given BI parameter $b$, both the HSC and HEE increase as the width $\ell$ increases. Furthermore, as shown in the Fig. 3, with increase of the BI parameter $b$ for the fixed belt width $\ell$ and temperature $T$, the HEE in the superconducting phase first increases and reaches the maximum value at some threshold, then decreases monotonically. This feature is the same as reported in [21]. However, we find that the dependence of HSC in the superconducting phase on BI parameters $b$ is also non-monotonic, but it is the opposite of the behavior of the HEE. As shown in the Fig. 4, for the fixed belt width $\ell$ and temperature $T$, the HSC in the superconducting phase first decreases and reaches its minimum at some threshold and then increases monotonically as the BI parameter $b$ increases. It should be noted that the BI parameter corresponding to the HSC reaching the inflection point is always greater than that of HEE. As pointed out in [53], the entanglement entropy is usually not enough to describe the rich geometric structure because it grows in a very short time during the thermalization process of a strongly coupled system. The information 

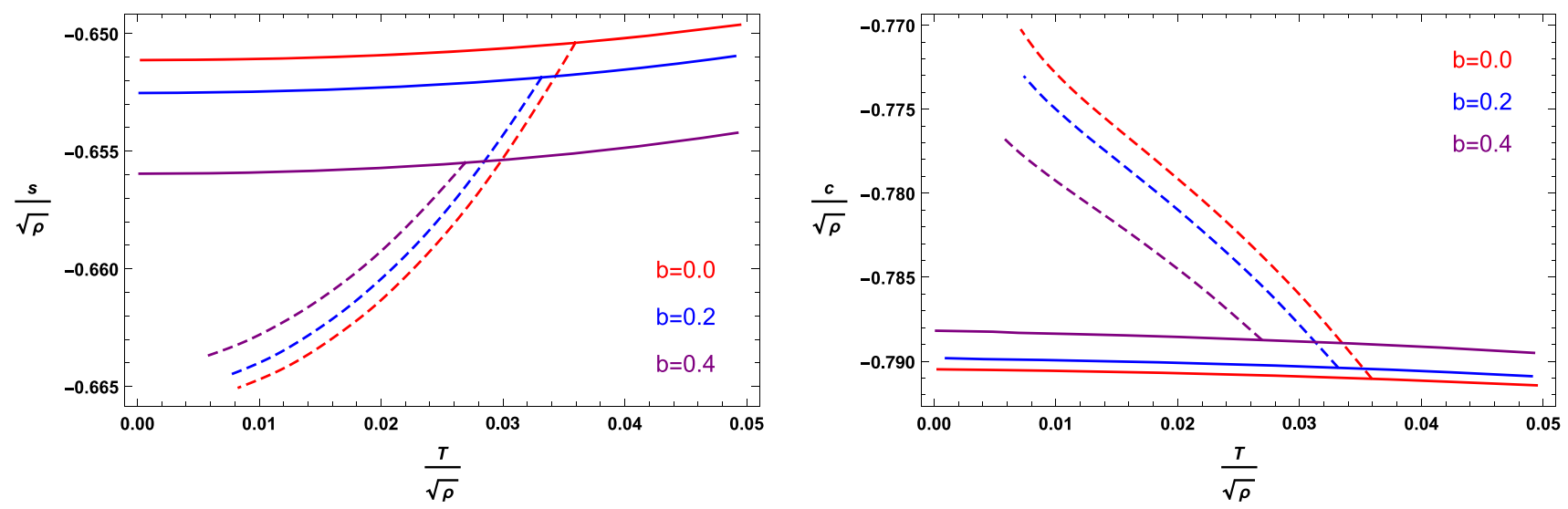

Fig. 2 The HEE (left) and HSC (right) of the $\mathcal{O}_{+}$as a functions of the temperature $T$ and BI factor $b$ for a fixed $\ell \sqrt{\rho}=1$. The solid and dashed curves indicate the normal and surperconducting phases. The colored curves correspond to $b=0$ (red), $b=0.2$ (blue) and $b=0.4$ (purple), respectively

reflected by the HSC may be different from the information captured by the HEE.

\subsection{The results of operator $\mathcal{O}_{-}$}

For the operator $\mathcal{O}_{-}$, we show the behaviors of HEE and HSC with respect to the temperature $T$ and BI parameter $b$ at a fixed strip width $\ell \sqrt{\rho}=1 \mathrm{in} \mathrm{Fig.} \mathrm{5.} \mathrm{The} \mathrm{left} \mathrm{panel} \mathrm{is} \mathrm{the}$ case of HEE and right panel shows the case of HSC. We find that both the HSC and HEE have discontinuous slopes near the same critical temperatures, which implies the non-trivial reorganization of the degrees of freedom in the system. This indicates that the system has undergone a phase transition from the normal state to the superconducting one. From the plot of HSC, we can see that the critical temperatures consistently drop when the BI parameter grows, which shows that the operator $\mathcal{O}_{-}$condenses more difficult when the BI electrodynamics correction becomes stronger. This result once again indicates that the HSC can be a possible probe of phase transition in the holographic superconducting system.

As can be seen from the left panel of Fig. 5, the HEE in the superconducting phase is always less than the one in the normal case and drops as the temperature decreases, which is consistent with the results of operator $\mathcal{O}_{+}$. This is reasonable because the cooper pairs form in the superconducting state which suppress of the degree of freedom of the system. From the right panel in Fig. 5, we can see that the HSC in the normal phase increases as the temperature decreases. Howerver, different from the results found in $\mathcal{O}_{+}$, the HSC in the superconducting phase drops as the temperature decreases and is always less than the one in the normal phase. This phenomenon does not only appear in this system. In fact, the HSC of two different operators also exhibit different behaviors in $2+1$ dimensional holographic superconductor, see [43], but the underlying mechanism remains mysterious.
To get further understanding of the effect of BI parameter on the HEE and HSC of the operator $\mathcal{O}_{-}$, we plot the corresponding results in Figs. 6 and 7 with a fixed temperature $T / \sqrt{\rho}=0.15$, which is below the transition temperature $T_{c}$. Obviously, for a given BI parameter $b$, both the HSC and HEE increase with the increase of the width $\ell$, which is consistent with the case of operator $\mathcal{O}_{+}$. We find that the behavior of HSC is quite similar to that of HEE in the superconducting phase, i.e., the effect of BI parameters on the HEE and HSC is monotonic, which is different from the case in the operator $\mathcal{O}_{+}$. For a fixed width $\ell$ (a given parameter $b$ ), the HSC and HEE increase monotonously with the increase of $\mathrm{BI}$ parameter $b$ (width $\ell$ ) in the superconducting phase.

\section{Summary and discussion}

In this paper, we conducted a numerical analysis of HSC and HEE in the metal/superconductor phase transition for the BI electrodynamics with full backreaction by using the subregion CV conjecture. We calculated the HSC of a strip subregion for two operators and compared it with the results of HEE. Our analysis shows that the HSC remains finite during phase transitions and the temperature where a normal phase turns into a superconducting phase is exactly the same in both the HSC and HEE computation, which corresponds exactly to the critical temperature $T_{c}$ of phase transition. Moreover, by calculating the HSC of the system, we noted that the critical temperature of the condensation for the operators becomes smaller with the increase of the BI parameter $b$, which implies that the stronger BI correction makes the scalar condensation harder to form. Our results indicates that the HSC can be a probe of phase transition in holographic superconductor models. 

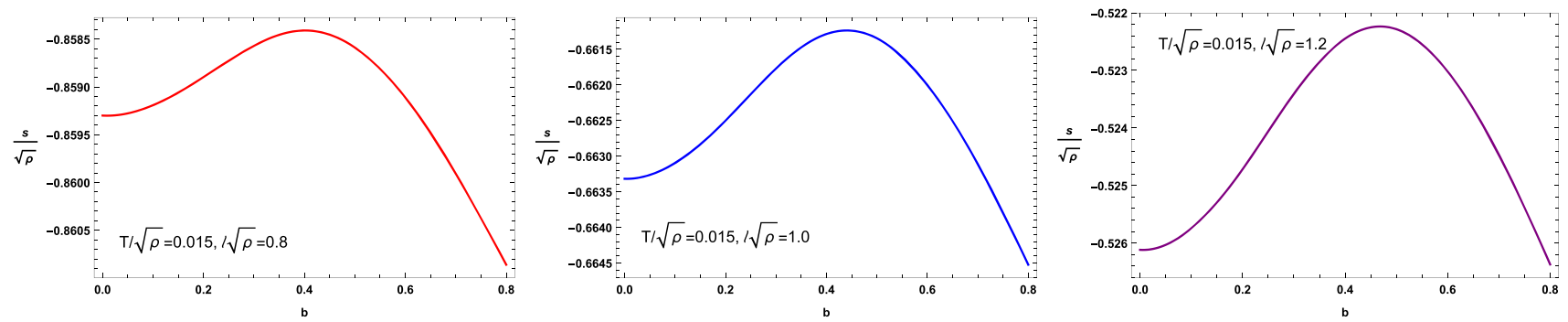

Fig. 3 The HEE of the operator $\mathcal{O}_{+}$as a function of the BI factor $b$ for different widths $\ell$ at $T / \sqrt{\rho}=0.015$. The left plot (red) is for $\ell \sqrt{\rho}=0.8$, the middle one (blue) for $\ell \sqrt{\rho}=1.0$, and the right one (black) for $\ell \sqrt{\rho}=1.2$
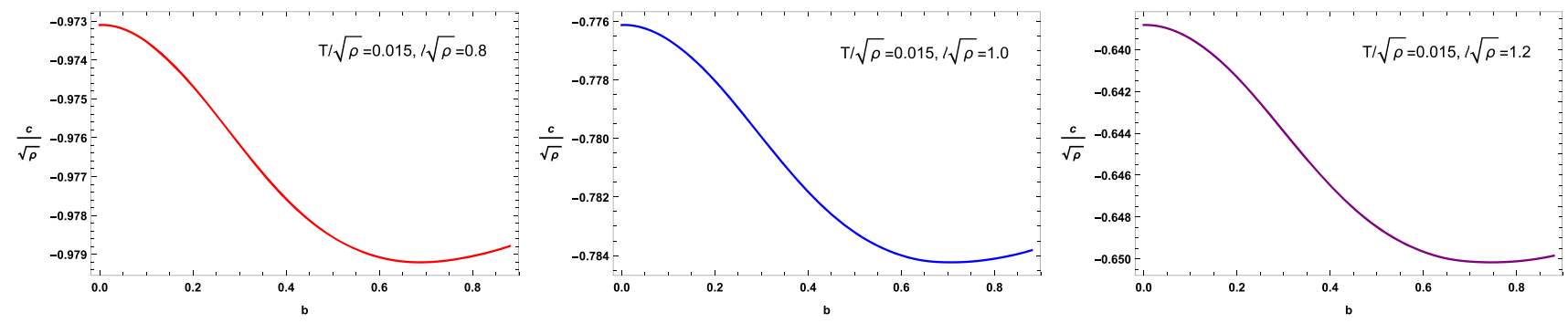

Fig. 4 The HSC of the operator $\mathcal{O}_{+}$as a function of the BI factor $b$ for different widths $\ell$ at $T / \sqrt{\rho}=0.015$. The left plot (red) is for $\ell \sqrt{\rho}=0.8$, the middle one (blue) for $\ell \sqrt{\rho}=1.0$, and the right one (purple) for $\ell \sqrt{\rho}=1.2$
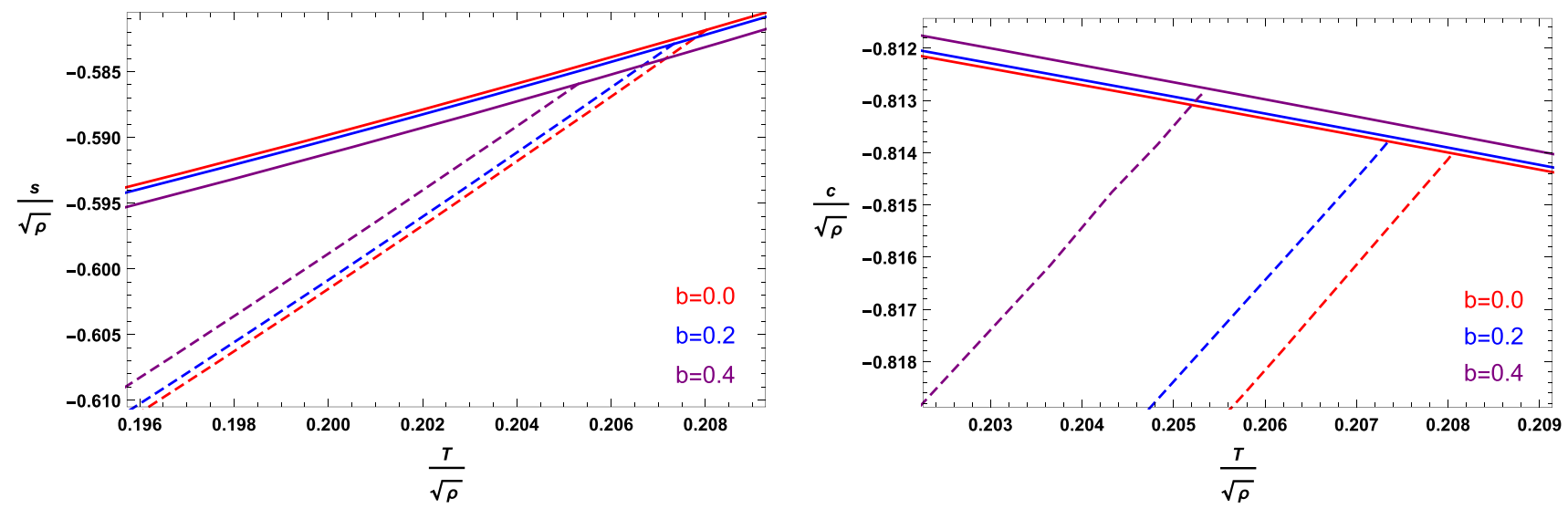

Fig. 5 The HEE (left) and HSC (right) of the $\mathcal{O}_{-}$as functions of the temperature $T$ and BI factor $b$ for a fixed $\ell \sqrt{\rho}=1$. The solid and dashed curves correspond to the normal and surperconducting phases, and the colored curves correspond to $b=0$ (red), $b=0.2$ (blue) and $b=0.4$ (purple), respectively
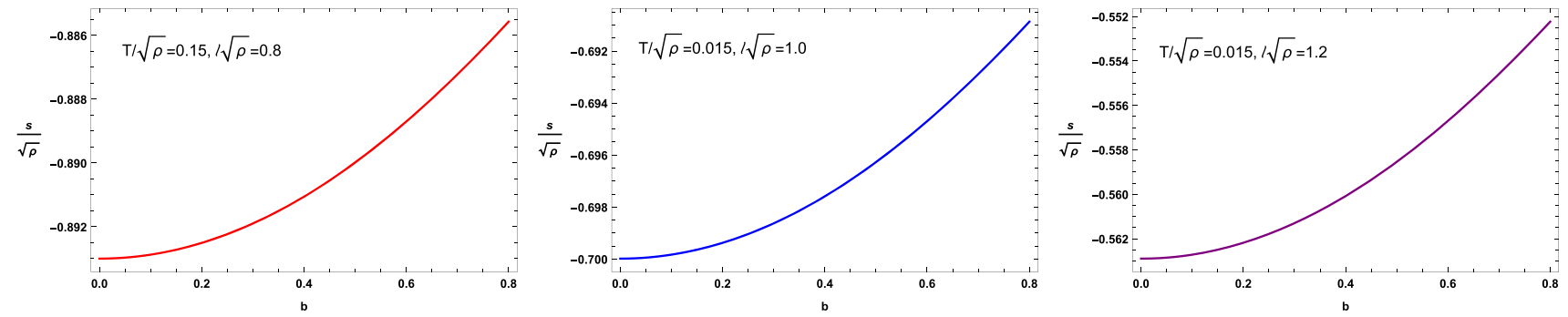

Fig. 6 The HEE of the operator $\mathcal{O}_{-}$as a function of the BI factor $b$ for different widths $\ell$ at $T / \sqrt{\rho}=0.15$. The left plot (red) is for $\ell \sqrt{\rho}=0.8$, the middle one (blue) for $\ell \sqrt{\rho}=1.0$, and the right one (purple) for $\ell \sqrt{\rho}=1.2$ 

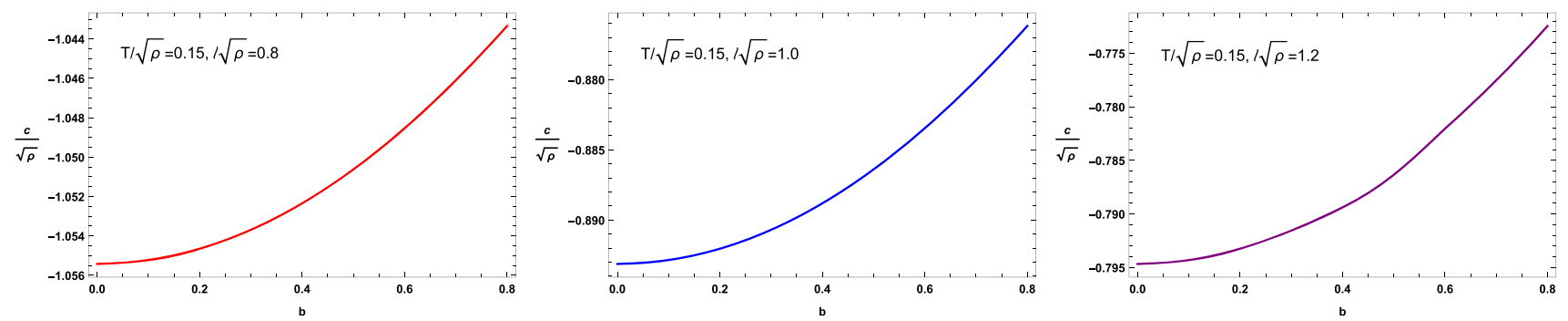

Fig. 7 The HSC of the operator $\mathcal{O}_{-}$as a function of the BI factor $b$ for different widths $\ell$ at $T / \sqrt{\rho}=0.15$. The left plot (red) is for $\ell \sqrt{\rho}=0.8$, the middle one (blue) for $\ell \sqrt{\rho}=1.0$, and the right one (purple) for $\ell \sqrt{\rho}=1.2$

It has been reported that the behavior of HSC mimics that of HEE for a thermodynamical phase transition of AdS black holes [54], but our results demonstrated that the HSC behaves in the different way with the HEE in the holographic superconducting phase transition. Concretely, for two operators, the HEE in the superconducting phase is always less than the one in the normal phase, and drops as the temperature decreases. This is due to the fact that the formation of Cooper pairs makes the degrees of freedom decrease in the superconducting phase. However, for the operator $\mathcal{O}_{+}$, we found that the HSC in the superconducting phase is always larger than the one in the normal phase, and increases as the temperature decreases. This result is in agreement with that reported in $[39,43]$. But for the operator $\mathcal{O}_{-}$, we found that the HSC in the superconducting phase is always less than the one in the normal phase. The HSC in the normal phase increases as the temperature decreases, while that in the superconducting phase is reversed. In other words, the behaviors of the HSC for two operators are also different. Similar phenomena have been found in Ref. [43]. This difference should, of course, not be confused with the difference caused by different orders of phase transition systems, but the underlying mechanism is still unclear. It does not contradict the results in [42], where the authors argued that the HSC can be a possible probe to the type of the superconducting phase transition. Because for the second order phase transition, its most notable feature is that, at the phase transition point the HSC is continuous and the slopes in terms of the temperature have a jump, which is consistent with our results. While in the first order superconducting phase transition, there exists the jump for the complexity at the critical temperature.

Furthermore, we found that the BI parameter $b$ has different effects on the HSC in the superconducting phase for the two operators. For the operator $\mathcal{O}_{-}$, the HSC increases monotonously with the increase of BI parameter, which is quite similar to that of HEE in the superconducting phase. Howerver, for the operator $\mathcal{O}_{+}$, the HSC in the superconducting phase first decreases to its minimum at some threshold and then increases as BI parameter $b$ increases, which is the opposite of the case of HEE. It should be noted that under the same conditions, the BI parameter corresponding to the
HSC reaching the inflection point is always greater than that of HEE. This implies that we may obtain richer physical information by using the HSC as a probe to study the phase transition. We noticed that, based on the definition of the subregion $\mathrm{CV}$ conjecture, the time evolution of HSC under a quench in the normal state has been studied in [55-59].

We note that the subregion complexity is deeply connected with the fidelity susceptibility. Therefore, studying the fidelity sensitivity of holographic superconducting systems from the dual field theory may provide a profound physical insights. In addition, since our results are based on the $\mathrm{CV}$ conjecture, an important question involved is whether the physical features we found depend on the chosen conjecture. Thus, it would be interesting to study the subregion complexity in the holographic superconducting system by using the CA conjecture. We will try to compute the complexity in the holographic superconducting model by using the CA conjecture and compare the results of the two conjectures in future work.

Acknowledgements This work was supported by the National Natural Science Foundation of China under Grant nos. 12035005 and 11875025 .

Data Availability Statement This manuscript has no associated data or the data will not be deposited. [Authors' comment: This is a theoretical study and no experimental data has been listed.]

Open Access This article is licensed under a Creative Commons Attribution 4.0 International License, which permits use, sharing, adaptation, distribution and reproduction in any medium or format, as long as you give appropriate credit to the original author(s) and the source, provide a link to the Creative Commons licence, and indicate if changes were made. The images or other third party material in this article are included in the article's Creative Commons licence, unless indicated otherwise in a credit line to the material. If material is not included in the article's Creative Commons licence and your intended use is not permitted by statutory regulation or exceeds the permitted use, you will need to obtain permission directly from the copyright holder. To view a copy of this licence, visit http://creativecomm ons.org/licenses/by/4.0/. Funded by $\mathrm{SCOAP}^{3}$. 


\section{References}

1. J.M. Maldacena, The large $\mathrm{N}$ limit of superconformal field theories andsupergravity. Adv. Theor. Math. Phys. 2, 231 (1998). arXiv:hep-th/9711200

2. S.S. Gubser, I.R. Klebanov, A.M. Polyakov, Gauge theory correlators from noncritical string theory. Phys. Lett. B 428, 105 (1998). arXiv:hep-th/9802109

3. E. Witten, Anti-de Sitter space and holography. Adv. Theor. Math. Phys. 2, 253 (1998). arXiv:hep-th/9802150

4. O. Aharony, S.S. Gubser, J.M. Maldacena, H. Ooguri, Y. Oz, Large$\mathrm{N}$ field theories, string theory and gravity. Phys. Rep. 323, 183 (2000). arXiv:hep-th/9905111

5. G.'t Hooft, Dimensional reduction in quantum gravity. Conf. Proc. C 930308, 284 (1993). arXiv:gr-qc/9310026

6. L. Susskind, The world as a hologram. Math. Phys. 36, 6377-6396 (1995). arXiv:hep-th/9409089

7. S.S. Gubser, Breaking an abelian gauge symmetry near a black hole horizon. Phys. Rev. D. 78, 065034 (2008)

8. S.A. Hartnoll, C.P. Herzog, G.T. Horowitz, Building a holographic superconductor. Phys. Rev. Lett. 101(2008), 031601 (2008). arXiv:hep-th/9409089

9. S.A. Hartnoll, C.P. Herzog, G.T. Horowitz, Holographic superconductors. J. High Energy Phys. 12, 015 (2008). arXiv:0810.1563

10. G.T. Horowitz, M.M. Roberts, Holographic superconductors with various condensates. Phys. Rev. D 78, 126008 (2008)

11. S. Ryu, T. Takayanagi, Entanglement entropy and the Berry phase in the solid state. Phys. Rev. Lett. 96, 181602 (2006)

12. S. Ryu, T. Takayanagi, Aspects of holographic entanglement entropy. J. High Energy Phys. 0608, 045 (2006)

13. G.T. Horowitz, Introduction to holographic superconductors. Lect. Notes Phys. 828, 313 (2011). arXiv: 1002.1722

14. R.G. Cai, L. Li, L.F. Li, R.Q. Yang, Introduction to holographic superconductor models. Sci. China Phys. Mech. Astron. 58(6), 060401 (2015). arXiv: 1502.00437

15. T. Albash, C.V. Johnson, Holographic studies of entanglement entropy in superconductors. J. High Energy Phys. 1205, 079 (2012). arXiv: 1202.2605

16. R.G. Cai, S. He, L. Li, Y.L. Zhang, Holographic entanglement entropy on p-wave superconductor phase transition. J. High Energy Phys. 1207, 027 (2012)

17. R.G. Cai, S. He, L. Li, Y.L. Zhang, Holographic entanglement entropy in insulator/superconductor transition. J. High Energy Phys. 1207, 088 (2012)

18. R.E. Arias, I.S. Landea, Backreacting p-wave superconductors. J. High Energy Phys. 1201, 157 (2013)

19. X.M. Kuang, E. Papantonopoulos, B. Wang, Entanglement entropy as a probe of the proximity effect in holographic superconductors. J. High Energy Phys. 05, 130 (2014)

20. W. Yao, J. Jing, Holographic entanglement entropy in insulator/superconductor transition with Born-Infeld electrodynamics. J. High Energy Phys. 05, 058 (2014)

21. W. Yao, J. Jing, Holographic entanglement entropy in metal/superconductor phase transition with Born-Infeld electrodynamics. Nucl. Phys. B 889, 109 (2014). arXiv:1408.1171

22. A. Dey, S. Mahapatra, T. Sarkar, Very general holographic superconductors and entanglement thermodynamics. J. High Energy Phys. 1412, 135 (2014). arXiv: 1409.5309

23. Y. Peng, Q. Pan, Holographic entanglement entropy in general holographic superconductor models. J. High Energy Phys. 1406, 011 (2014). arXiv:1404.1659

24. D. Momeni, H. Gholizade, M. Raza, R. Myrzakulov, Holographic entanglement entropy in 2D holographic superconductor via $A d S_{3} / C F T_{2}$. Phys. Lett. B 747, 417 (2015). arXiv:1503.02896
25. Y. Peng, Holographic entanglement entropy in superconductor phase transition with dark matter sector. Phys. Lett. B 750, 420 (2015). arXiv: 1507.07399

26. W. Yao, J. Jing, Holographic entanglement entropy in metal/superconductor phase transition with exponential nonlinear electrodynamics. Phys. Lett. B 759, 533 (2016). arXiv:1603.04516

27. Y. Ling, P. Liu, J.P. Wu, Characterization of quantum phase transition using holographic entanglement entropy. Phys. Rev. D 93(12), 126004 (2016). arXiv: 1604.04857

28. S.R. Das, M. Fujita, B.S. Kim, Holographic entanglement entropy of a 1+1 dimensional p-wave superconductor. J. High Energy Phys. 1709, 016 (2017). arXiv:1705.10392

29. W. Yao, C. Yang, J. Jing, Holographic insulator/superconductor transition with exponential nonlinear electrodynamics probed by entanglement entropy. Eur. Phys. J. C 78, 353 (2018)

30. L. Susskind, Computational complexity and black hole horizons. Fortsch. Phys. 64, 24 (2016). arXiv: 1403.5695

31. D. Stanford, L. Susskind, Complexity and shock wave geometries. Phys. Rev. D 90, 126007 (2014)

32. A.R. Brown, D.A. Roberts, L. Susskind, B. Swingle, Y. Zhao, Holographic complexity equals bulk action? Phys. Rev. Lett. 116, 191301 (2016)

33. A.R. Brown, D.A. Roberts, L. Susskind, B. Swingle, Y. Zhao, Complexity, action, and black holes. Phys. Rev. D 93, 086006 (2016)

34. M. Alishahiha, Holographic complexity. Phys. Rev. D 92, 126009 (2015)

35. M. Miyaji et al., Distance between quantum states and gaugegravity duality. Phys. Rev. Lett. 115, 261602 (2015)

36. W.C. Gan, F.W. Shu, Holographic complexity: a tool to probe the property of reduced fidelity susceptibility. Phys. Rev. D 96(2), 026008 (2017). arXiv:1702.07471

37. M. Flory, A complexity/fidelity susceptibility g-theorem for $A d S_{3} / C F T_{2}$. J. High Energy Phys. 1706, 131 (2017). arXiv: 1702.06386

38. D. Momeni, S.A.Hosseini Mansoori, R. Myrzakulov, Holographic complexity in gauge/string superconductors. Phys. Lett. B 756, 354 (2016). arXiv:1601.03011

39. M.Kord Zangeneh, Y.C. Ong, B. Wang, Entanglement entropy and complexity for one-dimensional holographic superconductors. Phys. Lett. B 717, 235 (2017). arXiv:1704.00557

40. R.Q. Yang, H.S. Jeong, C. Niu, K.Y. Kim, Complexity of holographic superconductors. J. High Energy Phys. 04, 146 (2019). arXiv: 1902.07586

41. A. Chakraborty, Holographic subregion complexity of a $1+1$ dimensional p-wave superconductor. Prog. Theor. Exp. Phys. 2019, 063B04 (2019). arXiv:1810.09659

42. H. Guo, X.M. Kuang, B. Wang, Note on holographic entanglement entropy and complexity in Stückelberg superconductor. Phys. Lett. B 797, 134879 (2019)

43. A. Chakraborty, On the complexity of a $2+1$-dimensional holographic superconductor. Class. Quantum Gravity 37, 065021 (2020). arXiv: 1903.00613

44. M. Born, L. Infeld, Foundations of the new field theory. Proc. R. Soc. A 144, 425 (1934)

45. G.W. Gibbons, D.A. Rasheed, Electric-magnetic duality rotations in non-linear electrodynamics. Nucl. Phys. 454, 185 (1995)

46. B. Hoffmann, Gravitational and electromagnetic mass in the BornInfeld electrodynamics. Phys. Rev. 47, 877 (1935)

47. J.L. Jing, S.B. Chen, Holographic superconductors in the BornInfeld electrodynamics. Phys. Lett. B 686, 68 (2010)

48. S. Gangopadhyay, D. Roychowdhury, Analytic study of properties of holographic superconductors in Born-Infeld electrodynamics. J. High Energy Phys. 05, 002 (2012)

49. P. Breitenlohner, D.Z. Freedman, Stability in gauged extended supergravity*. Ann. Phys. 144, 249 (1982) 
50. P. Breitenlohner, D.Z. Freedman, Positive energy in anti-de Sitter backgounds and gauged exrtended supergravity*. Phys. Lett. B 115, 197 (1982)

51. D. Carmi, R.C. Myers, P. Rath, Comments on holographic complexity. J. High Energy Phys. 03, 118 (2017). arXiv: 1612.00433

52. R.Q. Yang, C. Niu, K.Y. Kim, Surface counterterms and regularized holographic complexity. J. High Energy Phys. 09, 042 (2017). arXiv: 1701.03706

53. L. Susskind, Entanglement is not enough. Fortschr. Phys. 64, 49 (2016). arXiv: 1411.0690

54. P. Roy, T. Sarkar, Note on subregion holographic complexity. Phys. Rev. D 96, 026022 (2017). arXiv: 1701.05489

55. B. Chen, W.M. Li, R.Q. Yang, C.Y.Zhang, S.J. Zhang, Holographic subregion complexity under a thermal quench. J. High Energy Phys. 1807, 034 (2018). arXiv:1803.06680
56. Y. Ling, Y. Liu, C.Y. Zhang, Holographic subregion complexity in Einstein-Born-Infeld theory. Eur. Phys. J. C 79(3), 194 (2019). arXiv:1808.10169

57. Y.T. Zhou, M. Ghodrati, X.M. Kuang, J.P. Wu, Evolutions of entanglement and complexity after a thermal quench in massive gravity theory. Phys. Rev. D 100, 066003 (2019). arXiv:1907.08453

58. Y. Ling, Y. Liu, C. Niu, Y. Xiao, C.Y.Zhang, Holographic subregion complexity in general Vaidya geometry. J. High Energy Phys. 11, 034 (2019). arXiv:1908.06432

59. R. Auzzi, G. Nardelli, F.I.S. Massolo, G. Tallarita, On volume subregion complexity in Vaidya spacetime. J. High Energy Phys. 11, 098 (2019). arXiv:1908.10832 